\title{
Superconducting properties of $\mathrm{RuSr}_{2} \mathrm{GdCu}_{2} \mathrm{O}_{8}$ studied by SQUID magnetometry
}

\author{
Thomas P. Papageorgiou用 and Hans F. Braun \\ Physikalisches Institut, Universität Bayreuth, D-95440 Bayreuth, Germany \\ Thomas Herrmannsdörfer \\ Forschungszentrum Rossendorf, D-01314 Dresden, Germany
}

(Dated: October 24, 2018)

\begin{abstract}
For polycrystalline $\mathrm{RuSr}_{2} \mathrm{GdCu}_{2} \mathrm{O}_{8}(\mathrm{Ru}-1212)$, distinct peaks have been reported in d.c. magnetization in the superconducting state of the sample. $\mathrm{Sr}_{2} \mathrm{GdRuO}_{6}(\mathrm{Sr}-2116)$, the precursor for the preparation of $\mathrm{Ru}-1212$, shows similar peaks in the same temperature regime. Based on measurements performed on both bulk and powdered samples of Ru-1212 and Sr-2116, we exclude the possibility, that the observed behavior of the magnetization of Ru-1212 is due to Sr-2116 impurities. The effect is related to the superconductivity of $\mathrm{Ru}-1212$, but it is not an intrinsic property of this compound. We provide evidence that the observation of magnetization peaks in the superconducting state of Ru-1212 is due to flux motion generated by the movement of the sample in an inhomogeneous field, during the measurement in the SQUID magnetometer. We propose several tests, that help to decide, whether the features observed in a SQUID magnetization measurement of $\mathrm{Ru}-1212$ represent a property of the compound or not.

PACS numbers: 74.72.-h, 74.25.Ha, 75.50.-y
\end{abstract}

Keywords: superconductivity, SQUID magnetometry, $\mathrm{RuSr}_{2} \mathrm{GdCu}_{2} \mathrm{O}_{8}, \mathrm{Sr}_{2} \mathrm{GdRuO}_{6}$ 


\section{INTRODUCTION}

Since its first observation, high temperature superconductivity (HTSC) is a fascinating and very active field of research. The paper of J. G. Bednorz and K. A. Müllert triggered a race for higher superconducting transition temperatures, $T_{c}$, which led to the discovery of many new superconductors. All these compounds though, had a common feature: A layered structure, where the existence of $\mathrm{CuO}_{2}$ layers seemed to be essential for the observation of superconductivity, at least until recentlye. Because of this feature, the term Cuprates is very often used to describe the HTSC compounds and distinguish them from the elemental or more conventional superconductors described by the BCS theory ${ }^{3}$.

Although there is no complete theory explaining the superconductivity of the cuprates, the $\mathrm{CuO}_{2}$ layers are believed to be responsible for conductivity and superconductivity, while interspersed layers, either insulating or weakly metallic, act as charge reservoirs donating carriers to the $\mathrm{CuO}_{2}$ planes. Thus, the cuprates can be viewed as a stacking of superconducting sheets (consisting of the $\mathrm{CuO}_{2}$ layers) coupled by Josephson interaction.

There appears to exist a relation between the maximum $T_{c}$ of a cuprate and the number of the $\mathrm{CuO}_{2}$ layers per superconducting sheet. For example日, $\mathrm{La}_{2-x} \mathrm{M}_{x} \mathrm{CuO}_{4}$ with $\mathrm{M}=\mathrm{Ba}$, Sr, $\mathrm{Ca}$ and one $\mathrm{CuO}_{2}$ layer per superconducting sheet have a $T_{c}$ of about $30 \mathrm{~K}, \mathrm{RBa}_{2} \mathrm{Cu}_{2+m} \mathrm{O}_{6+m}$ with $\mathrm{R}=\mathrm{Y}$, La, Nd, Sm, Eu, Ho, Er, Tm, Lu and two $\mathrm{CuO}_{2}$ layers per superconducting sheet have a $T_{c}$ of the order of $90 \mathrm{~K}$, while $\mathrm{Bi}_{2} \mathrm{Sr}_{2} \mathrm{Ca}_{n-1} \mathrm{Cu}_{n} \mathrm{O}_{2 n+4}$ and $\mathrm{Tl}_{2} \mathrm{Ba}_{2} \mathrm{Ca}_{n-1} \mathrm{Cu}_{n} \mathrm{O}_{2 n+4}$ with $\mathrm{n}=3$ and three $\mathrm{CuO}_{2}$ layers per superconducting sheet have a $T_{c}$ of about 110 and $125 \mathrm{~K}$ respectively. Thus, the more $\mathrm{CuO}_{2}$ layers per superconducting sheet the higher the maximum $T_{c}$.

With this empirical relation in mind, it is interesting to investigate how the properties of a two-layer system, like $\mathrm{YBa}_{2} \mathrm{Cu}_{3} \mathrm{O}_{6+x}$ (YBCO), would be affected, if the coupling between the superconducting sheets is changed, with the introduction of a metallic block. An effort to follow this idea made by L. Bauernfeind国 led to the discovery of $\mathrm{RuSr}_{2} \mathrm{GdCu}_{2} \mathrm{O}_{8}(\mathrm{Ru}-1212)$ 6. where the $(\mathrm{Ba}, \mathrm{O})-(\mathrm{Cu}, \mathrm{O})-(\mathrm{Ba}, \mathrm{O})$ charge reservoir of $\mathrm{YBCO}$ is substituted by a $\mathrm{SrRuO}_{3}$-like block. $\mathrm{SrRuO}_{3}$ is a pseudocubic perovskite and a metallic itinerant ferromagnet with $T_{\text {Curie }}$ $\sim 160 \mathrm{~K}$.

The $T_{c}$ of Ru-1212 depends strongly on the preparation conditions 9 and there are reports

for non-superconducting samples 10 as well as for samples, in which the onset of supercon- 
ductivity reaches $50 \mathrm{~K}$ 月11 or even higher for $\mathrm{Ru} / \mathrm{Cu}$ substitutions 12 . In any case, it is low compared to that of YBCO, presumably because of the underdoped character of the $\mathrm{CuO}_{2}$ planes. Powder neutron diffraction studies 13.14 .15 showed, that the $\mathrm{Ru}$ (and Gd) moments in this compound order antiferromagnetically at $\sim 135$ (2.5) K. In more detail, a canted arrangement of the moments is indicated by hysteresis loops in d.c. magnetization vs. magnetic field measurements5.16, which reveal a ferromagnetic component in the compound's magnetic properties (weak ferromagnetism). The fact that Ru-1212 is magnetic, is not surprising, in view of the properties of $\mathrm{SrRuO}_{3}$ mentioned above, but makes the family of $\mathrm{Ru}$-1212 compounds 17, 18, 19, together with $\mathrm{RuSr}_{2}\left(\mathrm{R}_{0.7} \mathrm{Ce}_{0.3}\right)_{2} \mathrm{Cu}_{2} \mathrm{O}_{10}$ 2021,22,23, where $\mathrm{R}=\mathrm{Eu}$, Gd (Ru-1222), the only family of HTSC compounds, where superconductivity arises in a state, in which magnetic order is already developed.

There is some skepticism, especially whether superconductivity is a bulk property of Ru-1212, or even an intrinsic property of this compound at all. Xue et al.2 report the absence of a Meissner state for Ru-1212, while Chu et al.25 suggest the existence of a cryptosuperconducting structure in this compound. On the other hand, heat capacity26, together with Muon Spin Rotation 16 and Electron Spin Resonance experiments 27 indicate, that bulk superconductivity and magnetism in Ru-1212 coexist on a microscopic scale.

The skepticism, whether Ru-1212 is a bulk superconductor or not, is enhanced by the controversial results on the field cooled d.c. magnetization of Ru-1212 and related compounds published by different groups. Field expulsion shown in such a measurement, corresponding to a bulk Meissner effect, is generally considered as the key indicator for bulk superconductivity. Nevertheless, some published data include an increase of the magnetization at the temperature where intergranular coupling has been established 12 , sometimes followed by a decrease of the magnetization at lower temperatures, which leads to the appearance of a peak in the magnetization vs. temperature plot.28. Klamut et al.29 have tentatively attributed these features to a change of the magnetic ordering of the $\mathrm{Ru}$ sublattice upon entering the superconducting state or to an anomalous flux lattice behavior. Artini et al 30 and Bauernfeind $\mathrm{g}$ report a rather "reversed" effect, where a decrease of the magnetization is observed first, attributed to a Meissner behavior, followed by an increase of the magnetization at lower temperatures. Artini et al.30 attribute the poor visibility of the Meissner state to the existence of a spontaneous vortex state proposed by Bernhard et al 11 or to a phase-lock of an aggregation of small Josephson-coupled superconducting grains or domains 
proposed by Chen et al.22. The field cooled curve of Klamut et al.29, with an increase of the magnetization just below $T_{c}$, followed by a plateau at low temperatures is also reminiscent of a "reversed" effect compared to the field cooled curves published by Bernhard et al.11, which show a decrease of the magnetization just below $T_{c}$ and a plateau at low temperatures, considered as evidence for the existence of a bulk Meissner state in Ru-1212.

In their paper, Artini et al. 30 recognize that the complexity of the magnetic signal of $\mathrm{Ru}-1212$, which consists of contributions from the $\mathrm{Gd}$ paramagnetic spin lattice, the $\mathrm{Ru}$ spin lattice and the diamagnetic signal due to superconductivity, can drastically affect the quality of a SQUID magnetization measurement, if this is done in a non-uniform field. Indeed, the different behaviors of the field cooled d.c. magnetization of Ru-1212 described above are reminiscent of features reported by McElfresh et al.31 for an YBCO film measured in different (measured) field profiles. These features though, the specific characteristics of which depended on the profile of the measuring field, did not represent intrinsic properties of the sample. They did arise from the fact, that the magnetization of the sample was changing, because of the non-uniform field, during a measurement at fixed temperature, while the algorithms used by the magnetometer's software to calculate the magnetization assume that this does not happen. It is the purpose of this paper to investigate whether Ru-1212 shows a similar sensitivity to field inhomogeneities, which could give rise to experimental artefacts, during a SQUID d.c. magnetization measurement.

\section{EXPERIMENTAL}

\section{A. Sample preparation and characterization}

Polycrystalline samples of Ru-1212 were prepared following a two-step procedure proposed by Bauernfeind et al. of $\mathrm{RuO}_{2}, \mathrm{Gd}_{2} \mathrm{O}_{3}$ and $\mathrm{SrCO}_{3}$. The mixed powders were ground, calcined at $950{ }^{\circ} \mathrm{C}$ in air, reground, milled, pressed into pellets and fired for $16 \mathrm{~h}$ at $1250{ }^{\circ} \mathrm{C}$ in air. In a second step, the obtained Sr-2116 was mixed with $\mathrm{CuO}$ and the mixture was ground, milled, pressed into pellets and fired for $120 \mathrm{~h}$ at $1060{ }^{\circ} \mathrm{C}$ in flowing oxygen.

A Seifert XRD 3000 P diffractometer was used for the sample characterization. The powder diffraction data were recorded for 40 seconds at each $2 \theta$ in steps of $0.01^{\circ}$ from $5^{\circ}$ to 
$75^{\circ}$. A weak peak indicative of $\mathrm{SrRuO}_{3}$ trace impurities was detected in the pattern of the sample.

\section{B. Measurements}

Resistance measurements were performed with a standard four-probe a.c. technique (at $22.2 \mathrm{~Hz}$ ) on bar-shaped pieces cut from the pellets using silver paint contacts.

a.c. susceptibility measurements were done with a home-made susceptometer using a standard lock-in technique at $22.2 \mathrm{~Hz}$ with different field amplitudes.

d.c. magnetization measurements were done with a commercial SQUID magnetometer (Cryogenic Consultants Ltd. S600) in the temperature range $7 \mathrm{~K} \leq T \leq 200 \mathrm{~K}$ and magnetic fields $B<10 \mathrm{mT}$. In order to overcome the problem of remanent fields, we used paramagnetic samples with high magnetic moments in low fields (e.g. $\mathrm{PrCu}_{6}$ ) as field sensors. The magnet power supply was disconnected and an external current source (Knick DC-Current-Calibrator J152) was used to apply the appropriate current for the cancellation of the remanent field according to the signal from the paramagnetic sample. Complete cancelation is difficult to be achieved, but after this procedure, values for the magnetic moment of the paramagnetic samples close to the resolution of our SQUID $\left(5 \cdot 10^{-10} \mathrm{Am}^{2}\right)$ were recorded at $7 \mathrm{~K}$. Comparing this signal with that at the same temperature in a field of $92.9 \mathrm{mT}$ we estimate a remanent field of $1.5 \mu \mathrm{T}$. The low fields of figures 2 and 3 were also determined by a comparison of the paramagnetic sample's signal at $7 \mathrm{~K}$ with that at the same temperature in a field of $92.9 \mathrm{mT}$. Nevertheless, since our measurements indicate that field inhomogeneties were present, the given field values should be considered as estimates. For this reason, when a comparison between measurements in a certain field was necessary, the samples were measured one after the other, before any change of the field was undertaken.

\section{RESULTS AND DISCUSSION}

\section{A. Superconductivity and magnetism of our Ru-1212 sample}

Figure 11 and figure 2 show the a.c. susceptibility, resistivity, as well as the zero field cooled (z.f.c) and field cooled (f.c.) d.c. magnetization curves of our Ru-1212 sample. A 

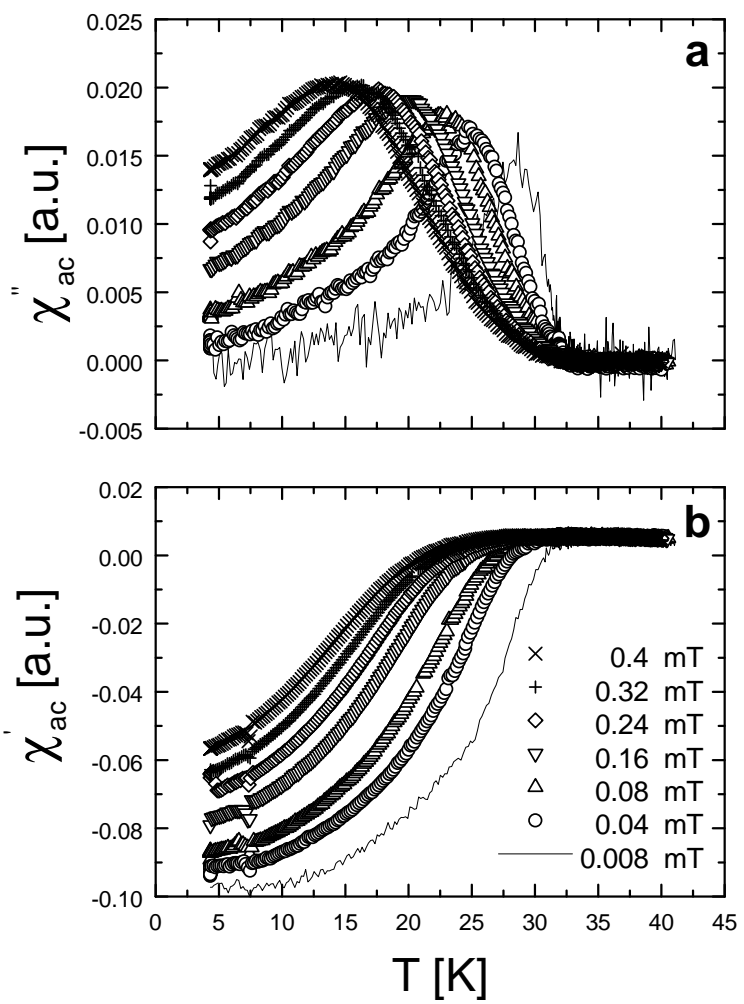

FIG. 1: The low temperature behavior of (a) the imaginary and (b) the real part of the a.c. susceptibility of our Ru-1212 sample for different field amplitudes.

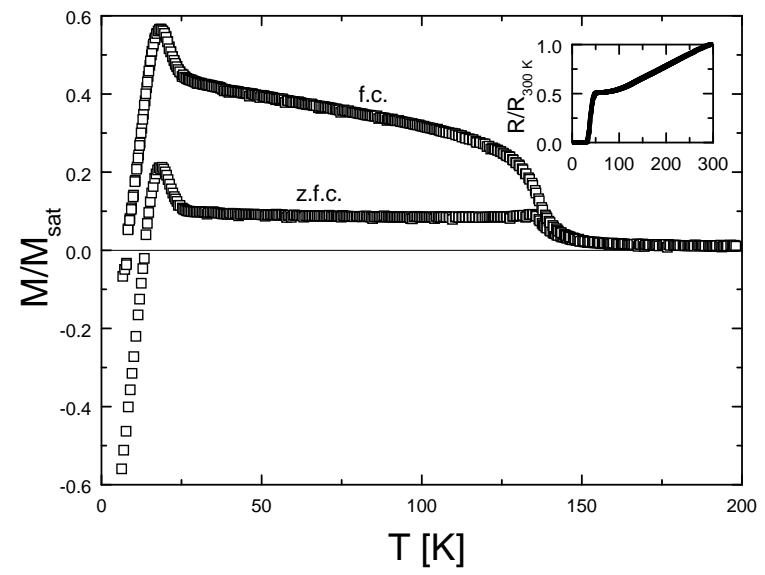

FIG. 2: d.c. magnetization of our Ru-1212 sample measured in a field of $0.25 \mathrm{mT}$. The magnetic moment $\mathrm{M}$ is normalised to the value of the magnetic moment $\mathrm{M}_{\text {sat }}$ corresponding to complete flux expulsion from the sample. Inset: Resistance of the same sample normalised to the room temperature value. 
magnetic transition is observed, as expected 13, 14, 15, at $135 \mathrm{~K}$. The hysteresis between the z.f.c. and f.c. branch of the d.c magnetization probably arises from the canting of the antiferromagnetically ordered $\mathrm{Ru}$ moments 10 . Increased canting of the $\mathrm{Ru}$ moments due to the presence of the external magnetic field in the f.c. process leads to higher values of the magnetization compared to the z.f.c. branch. The resistance of the sample, which has a metallic behavior at high temperatures, shows a cusp in the temperature range of the magnetic transition. The onset of superconductivity is at $50 \mathrm{~K}$, while the resistance becomes zero at $30 \mathrm{~K}$. At this temperature, the inter-granular coupling is established and a clear diamagnetic response is observed in the real part of the a.c. susceptibility with the corresponding loss peaks in the imaginary part. Typical for shielding due to inter-granular coupling, the transition widens and shifts to lower temperatures, as the a.c. field amplitude is increased 32 .

Distinct anomalies of the d.c. magnetization are observed for our Ru-1212 sample as it enters the superconducting state. The curves presented in figure 2 show a clear increase at $25 \mathrm{~K}$ and a peak at a temperature of $18 \mathrm{~K}$, below which a magnetization decrease indicative of field expulsion due to superconductivity begins. Klamut et al. 29 were, to the best of our knowledge, first to report similar features. Their sample had a higher $T_{c}$ of $35 \mathrm{~K}$ and the peak was observed above $25 \mathrm{~K}$ under the condition of zero field cooling. The origin of these peaks is still unclear. A comparison of figure 1 with figure 2 shows, that the onset of the peaks $(\sim 25 \mathrm{~K})$ is close to the temperature, where intergranular coupling has been established. Thus, the increase of the magnetization happens when shielding currents start to flow between the grains. This fact was also observed by Klamut et al.20.

Figure 3 shows the behavior of the low temperature Ru-1212 magnetization peak in different magnetic fields. The position of the peak $(\sim 18 \mathrm{~K})$ does not change significantly with magnetic field up to $3.6 \mathrm{mT}$. Above this field value, the feature is washed out and the Gd paramagnetic contribution seems to dominate at low temperatures. In these higher fields the Gd contribution becomes more significant also at higher temperatures, in the normal state of the sample.

In the following, we will investigate the origin of these features. We will examine first though, whether the observed behavior of the Ru-1212 magnetization at low temperatures is not related to impurities. 


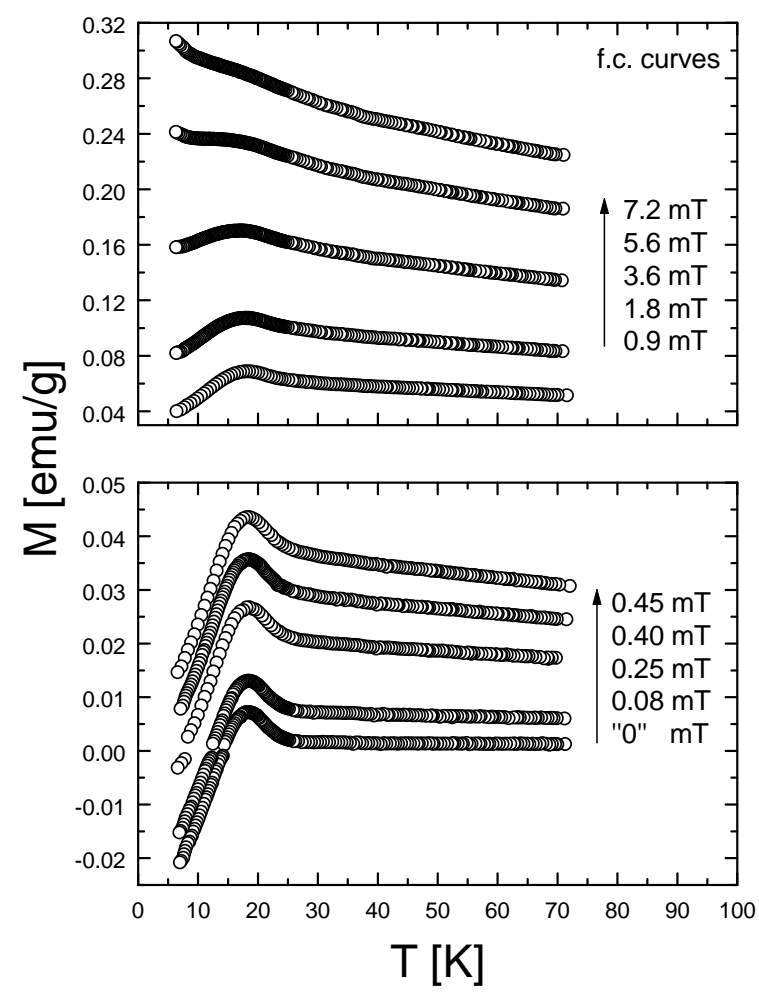

FIG. 3: Low temperature field-cooled d.c. magnetization of the Ru-1212 sample of figure 2 in different magnetic fields. The "0" field is the tiny remanent field, which was achived using the $\mathrm{PrCu}_{6}$ sample as a field sensor, following the method described in section II.

\section{B. Can the peaks be due to $\mathrm{Sr}_{2} \mathrm{GdRuO}_{6}$ trace impurities ?}

The d.c. magnetization of $\mathrm{Sr}_{2} \mathrm{GdRuO}_{6}(\mathrm{Sr}-2116)$ is shown in figure 4. Sr-2116 can appear as an impurity phase in the Ru-1212 matrix and in cases like ours, is used as a precursor for the preparation of Ru-1212 of the $\mathrm{Ru}-1212$ preparation. In the X-ray pattern traces of the original $\mathrm{Gd}_{2} \mathrm{O}_{3}$ powder were still present. The specific features of the Sr-2116 magnetization were presented and analysed in our previous work 28 and will not be discussed here. It is interesting to note, that Sr-2116 shows a magnetization peak around $18 \mathrm{~K}$, i.e., in the temperature range, where the peaks for Ru-1212 are observed.

In figure पla, measurements on bulk samples of Ru-1212 and Sr-2116 are shown. The results for $\mathrm{Ru}-1212$ presented in figure ta and those in the previous figures are on two different pieces of Ru-1212 coming from the same pellet. All the necessary quantities for a 


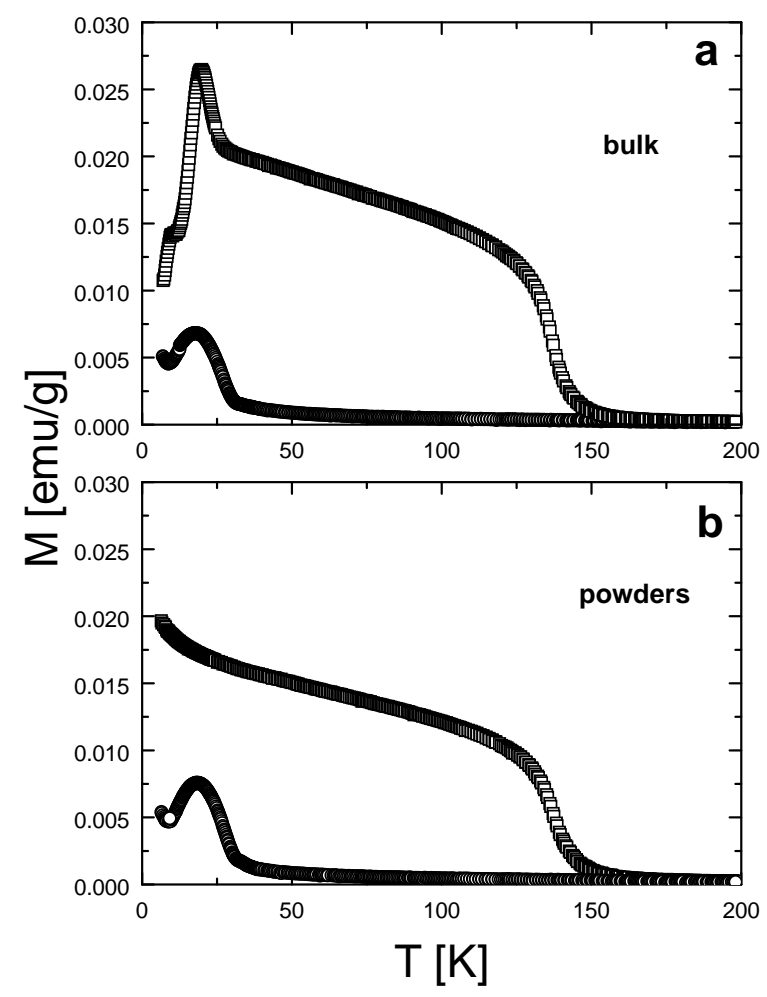

FIG. 4: (a) Field-cooled d.c. magnetization of bulk Ru-1212 (open squares) and Sr-2116 (open circles). The size of the peak for Ru-1212 in units of magnetic moment was $6.2 \times 10^{-8} \mathrm{Am}^{2}$ and the mass of the sample was $10.12 \mathrm{mg}$. For Sr-2116 the size of the peak was $9 \times 10^{-8} \mathrm{Am}^{2}$ and the mass of the sample was $14.14 \mathrm{mg}$. (b) The same after the two samples presented in (a) were powdered. The measuring field was $0.25 \mathrm{mT}$ in all cases.

comparison between the two compounds are given in the figure caption: the height of the peaks in units of magnetic moment and the masses of the samples. Although our X-ray data put an upper limit of about $0.3 \mathrm{mg}$ to possible Sr-2116 impurities in our Ru-1212 sample, which represents a concentration of about $3 \%$ (resolution of the instrument), it is obvious, that $\sim 9 \mathrm{mg}$, or $88 \%$, of Sr-2116 impurities would be needed to quantitatively explain the magnetization peak of the Ru-1212 sample as arising due to $\mathrm{Sr}-2116$.

Similar argumentation was used in our previous work 28 to exclude the possibility that the peaks observed for Ru-1212 are due to Sr-2116 impurities. The fact though, that possible Sr-2116 grains are enclosed in a magnetic Ru-1212 matrix was not taken into account. We do not expect, that the magnetism of Ru-1212 would affect the behavior of Sr-2116 significantly when the sample is cooled in a small applied field of $0.25 \mathrm{mT}$, like in the case presented in 
figure 4 . Assuming a homogeneous Ru-1212 matrix and using the magnetization M at $25 \mathrm{~K}$ for the bulk Ru-1212 sample from figure Æa, we estimate a contribution to the magnetic field from the Ru-1212 compound $\mathrm{B}=\mu_{0} \cdot \mathrm{M}=0.2 \mathrm{mT}$, which could not enhance the magnetism of small amounts of Sr-2116, not visible in X-ray powder diffraction patterns, distributed in the Ru-1212 matrix to the level of the Ru-1212 peaks.

We have already discussed 28 how parameters, which can be affected during the preparation of Sr-2116, like oxygen content, $\mathrm{Ru}$ deficiencies or $\mathrm{Cu}$ doping (this could take place for example during the preparation of $\mathrm{Ru}-1212$ by adding $\mathrm{CuO}$ to $\mathrm{Sr}-2116$ ), could enhance the magnetism of Sr-2116 and then smaller amounts of this compound would be necessary to produce the peaks measured for $\mathrm{Ru}-1212$. Indeed, the $\mathrm{Sr}-2116$ peaks we found previously 22 were more pronounced compared to those of figure $₫$. These points make the above quantitative comparison somewhat uncertain. However, in none of the cases we studied 28 , did we find a peak for Sr-2116-like compounds, which could explain the Ru-1212 peak, assuming an impurity level consistent with our X-ray data.

In order to further investigate whether the magnetization anomalies in the superconducting state of Ru-1212 are due to Sr-2116 impurities or not, we powdered the samples of Ru-1212 and Sr-2116 of figure tha and remeasured their magnetization. The powders were embeded in GE varnish for the measurements. The result is shown in figure thb. The powdered Ru-1212 sample has a completely different behavior at low temperatures. The peak has dissappeared together with the magnetization decrease indicative of field expulsion due to superconductivity. This behavior can be attributed to grain size of the order of the penetration depth or to the quasi-two-dimensional character of the superconducting regions 12 which prevents intragrain flux expulsion to occur. On the other hand, the properties of Sr-2116 remain unchanged after powdering. Thus, if the Ru-1212 peak was due to Sr-2116 impurities, we expect it to still be present in the powdered sample.

A further argument is that the position of the $\mathrm{Ru}-1212$ peaks is not fixed for all samples and seems to follow the temperature, at which intergranular coupling is established. In another case, where the Ru-1212 magnetization peaks have been observed 29, the sample had a higher $T_{c}$ of $35 \mathrm{~K}$ compared to ours and the peak was observed above $25 \mathrm{~K}$. In this case it would be very difficult to attribute the observed peak to Sr-2116 impurities, not only quantitatively, like in our case, but also as far as the peak temperature is concerned.

The above analysis indicates, that the magnetization peaks observed in the supercon- 
ducting state of Ru-1212 are related to the superconductivity of this compound. In the following we will show though, that they are not an intrinsic property of this compound, but arise from the movement of the sample in a non-homogeneous field during the measurement with the SQUID magnetometer. Nevertheless, we begin by discussing a model for the explanation of these peaks, as if they represented an intrinsic property of the compound. This discussion will (i) help us illustrate clearly the danger of developing impressive, but invalid, explanations, when a careful check of the SQUID magnetization of Ru-1212 has not been done and (ii) serve as the starting point for the proposal and evaluation of several tests by which the validity of the magnetization features calculated by the SQUID's software for Ru-1212 (or any other superconductor) can be investigated.

\section{A possible (but not real) origin of the Ru-1212 magnetization peaks}

Recently, the occurrence of the Paramagnetic Meissner Effect (PME) has been predicted for superconductors, when they are cooled in a field below $T_{c}$, from the self-consistent solution of the Ginzburg-Landau equation 33 . The physical picture behind this model is as

follow 33 : when a vortex is present inside a superconductor, the current around it flows in a direction to screen the vortex field from entering the bulk of the sample. In a magnetic field, additional surface current flows in order to prevent the field from entering the interior of the superconductor. These two currents flow in opposite directions and contribute with different signs to the superconductor's magnetization M. The current around the vortices gives a positive (paramagnetic) contribution, while the surface current gives a negative (diamagnetic) contribution. The resulting value of $\mathrm{M}$ can be negative or positive depending on the value of the magnetic field. The PME arises then, from the imbalance between the two screening currents. This physical picture can explain the magnetization peaks observed for our Ru-1212 samples as the result of the competition between the screening currents around the vortices, which dominates at temperatures close to $T_{c}$, and the surface current, the diamagnetic contribution of which starts to dominate at lower temperatures.

The model predicts the existence of vortex states with different vorticities, each of which exists in a certain range of magnetic fields. Some of these states are paramagnetic and some diamagnetic. Equilibrium transitions between them do not allow the observation of the PME. On the other hand, if metastable states exist, introduced by vortex pinning, the 


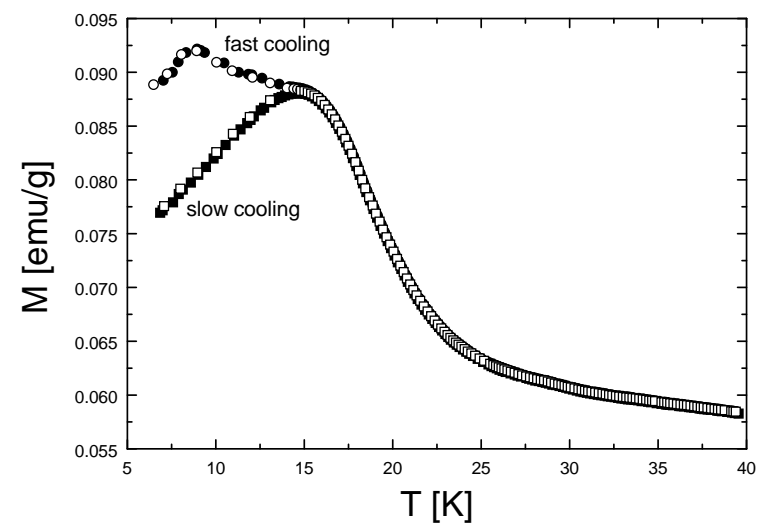

FIG. 5: f.c. d.c. magnetization of bulk Ru-1212 using two different cooling rates. The closed and open squares represent the two measurements under slow cooling conditions, while the closed and open circles the two measurements under fast cooling conditions (see text). For clarity several data points have been deliberately omitted below the peak position. The measuring field was $1 \mathrm{mT}$.

observation of the PME is possible. In order to investigate this feature of the model, we did f.c. d.c. magnetization measurements using different cooling rates. The result is shown in figure 5. The slow cooling measurement, which showed the peak, was done by cooling the sample from $200 \mathrm{~K}$ with a cooling rate of about $0.2 \mathrm{~K} / \mathrm{min}$ below $40 \mathrm{~K}$. The cooling rate was controlled by taking the measurements during cooldown (similar to the procedure followed for the curves of figure 3) with a step of $0.2 \mathrm{~K}$ from 40 to $7 \mathrm{~K}$. The curve was remeasured during warm up and no hysteresis was observed. The fast cooling measurement was done by cooling the sample directly from $200 \mathrm{~K}$ to $7 \mathrm{~K}$ within 2 hours. The measurements were taken during warm up. The difference between the two curves is obvious below the peak position of the slow cooling measurement. The fast cooling measurement was repeated under the same cooling conditions. Now the measurements were not taken immediately after reaching $7 \mathrm{~K}$, but after the sample was left at $7 \mathrm{~K}$ for 24 hours. This second measurement, as shown in figure 5, was identical with the first one under fast cooling conditions. After this second fast cooling measurement, the slow cooling measurement was also repeated and it gave the same result as the first slow cooling measurement. The observed dependence of the measured magnetization on the cooling rate is in accordance with the model's prediction, that the observation of the PME is a signal of metastability. At this point we should note, that considerations similar to those developed here were recently used to introduce the PME in 
$\mathrm{MgB}_{2}$ B 3 . There the peak position, where the curves measured under different cooling rates merge, was identified as the irreversibility temperature connected with the vortex pinning that introduces metastability.

According to Zharkov33, the appearance of the PME is determined by the size parameter $\mathrm{A}=(\mathrm{R} / \kappa)\left(2 \pi \mathrm{H} / \Phi_{0}\right)^{1 / 2}$, where $\mathrm{R}$ is the diameter of the sample, $\kappa$ is the Ginzburg-Landau parameter, $\mathrm{H}$ the magnetic field and $\Phi_{0}$ the flux quantum. For $\mathrm{A}=1$, for example, no PME is expected 33 , while for $\mathrm{A}=3$ the appearance of the PME is allowed 33 . The dependence of the PME appearance on the parameter A can explain why intergranular coupling has to be established, before the PME is observed. First of all, for samples with grain size of the order of the penetration depth or smaller, the establishment of intergranular coupling is the only possibility for the creation of the surface current, which creates the diamagnetic contribution to M. On the other hand, for samples with grain size bigger than the penetration depth, if this size gives a value of A not consistent with the appearance of the PME, then the effect will not be observed before intergranular coupling is established. So, intergranular coupling changes the characteristic size of the sample and can lead from A values not consistent with the appearance of the PME to values consistent with it.

In the study of $\mathrm{MgB}_{2}$, Horvat et al 34 recognize that the appearance of the PME in the z.f.c. data is difficult to understand. They consider this a feature, which distinguishes the PME reported for $\mathrm{MgB}_{2}$ from the $\mathrm{PME}$ in the conventional or high temperature superconductors. For Ru-1212 the appearance of the PME in the z.f.c. curve (see z.f.c. data in figure 2) can be understood if one keeps in mind, that Ru-1212 is a magnetic superconductor and even when it is cooled in zero magnetic field, it will react to its own magnetism. An indication for this is given by the measurement in "0" field presented in figure 3, although the existence of a tiny remanent field can not be avoided. Since within the framework of the model presented a vortex state is necessary for the observation of the PME, the observation of the magnetization peak in this curve also can be considered indicative of the existence of the spontaneous vortex phase in $\mathrm{Ru}-1212$, as has been already discussed for the Ruthenium-cuprates11.35. 


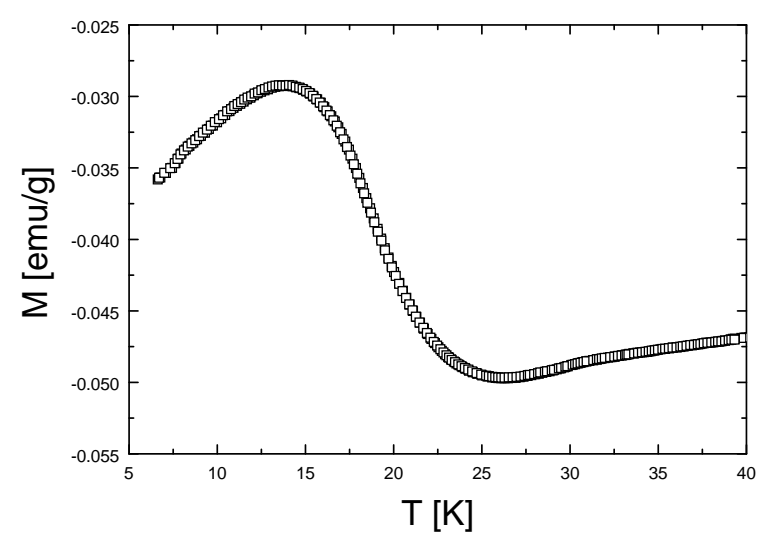

FIG. 6: The f.c. d.c. magnetization measurement of figure 5 with inversed field direction.

\section{The real origin of the Ru-1212 magnetization peaks}

In section $\amalg I I C$ we proposed a model for the explanation of the d.c. magnetization measurements on our Ru-1212 sample, which practically explains all the experimental observations. Within this model, the observed features in the magnetization of our $\mathrm{Ru}-1212$ sample were explained as arising from the competition of a paramagnetic contribution from the currents around the vortices and a diamagnetic contribution from the surface current. If this is the case, we expect that a reversal of the field should lead to a reversal of the observed features. In figure 6, we show the slow cooling measurement of figure 5 in a field of $-1 \mathrm{mT}$. While the paramagnetic signal observed above $30 \mathrm{~K}$ is reversed as expected, the peak is observed again but it has not been reversed by the inversion of the field. This fact indicates, that the mechanism proposed in section $\mathbb{\mathrm { IIC }}$ for the explanation of the magnetization peaks observed in the superconducting state of $\mathrm{Ru}-1212$ is not the appropriate one.

In order to further investigate the validity of our assumptions in section 【IIQ, we have tested the reproducibility of our measurements after the superconducting magnet of the magnetometer was cycled in the following way: $0 \mathrm{~T} \rightarrow 6 \mathrm{~T} \rightarrow-6 \mathrm{~T} \rightarrow 0 \mathrm{~T} \rightarrow 0.05 \mathrm{mT}$. The f.c. measurement after this cycle is shown in figure 0. It is obvious that the effect has reversed sign compared to figure 2. Now a decrease of the magnetization is observed below $T_{c}$ followed by a rapid increase of the magnetization at lower temperatures.

The result presented in figure 6 is similar to an observation made by Blunt et al. 3 , when they investigated the origin of "paramagnetic" moments in the superconducting state of $\left(\mathrm{Tl}_{0.5} \mathrm{~V}_{0.5}\right) \mathrm{Sr}_{2}\left(\mathrm{Ca}_{0.8} \mathrm{Y}_{0.2}\right) \mathrm{Cu}_{2} \mathrm{O}_{y}$. On the other hand, the result of figure 7 is reminiscent of 


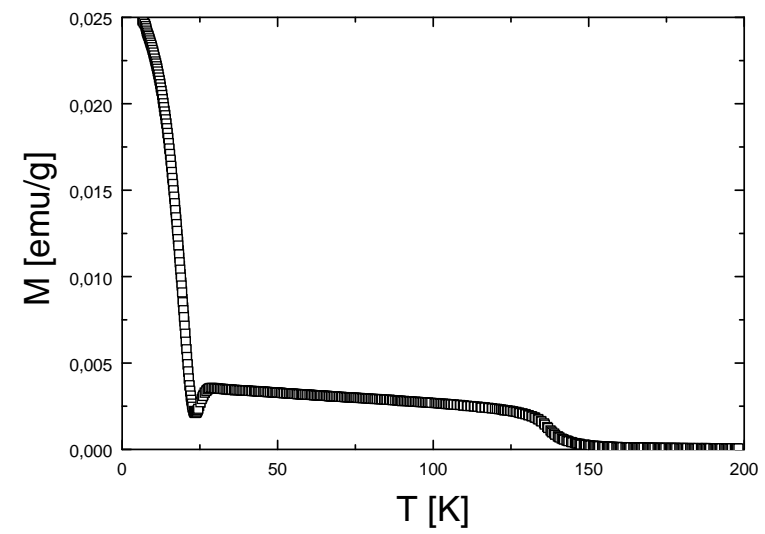

FIG. 7: f.c. d.c. magnetization measurement of our Ru-1212 sample in a field of $0.05 \mathrm{mT}$ after the cycle $0 \mathrm{~T} \rightarrow 6 \mathrm{~T} \rightarrow-6 \mathrm{~T} \rightarrow 0 \mathrm{~T} \rightarrow 0.05 \mathrm{mT}$.

effects reported by McElfresh et al. 31 for an YBCO thin film, where field profiles symmetric with respect to a set value of the magnetic field created magnetization features with reversed signs in the superconducting state of the sample. We note, that the cycling of the magnet to high fields, before the measurement of figure 7, is very likely to have changed the profile of the field compared to that of measurements presented in previous figures 31 .

In both cases reported above, the authors 31.36 have interpreted their observations as artefacts arising from the movement of the sample in a non-homogeneous field during the measurement with the SQUID magnetometer. The problem is as follows: For many of the commercially available magnetometers the measurement requires the motion of the sample through a pickup coil system. These coils are wound in a second derivative configuration, where the two outer detection loops, located at a distance A from the center of the magnetometer's magnet, are wound oppositely to the two central loops located at the center of the magnet. During the measurement, the movement of the sample through the pickup coils induces currents in the detection loops, which, through an inductance L, create magnetic flux in the SQUID circuit, resulting in an output voltage $\mathrm{V}$, which depends on the position of the sample $z$. This signal V(z) is fitted by the SQUID's software for the determination of the sample's magnetic moment. Nearly all analysis methods of the $\mathrm{V}(\mathrm{z})$ signal make two significant assumptions for the magnetic moment of the sample: (a) that it is approximated by a magnetic dipole moment and (b) that the sign and value of this moment do not change during the measurement. A superconducting sample though, will follow a minor hysteresis loop 

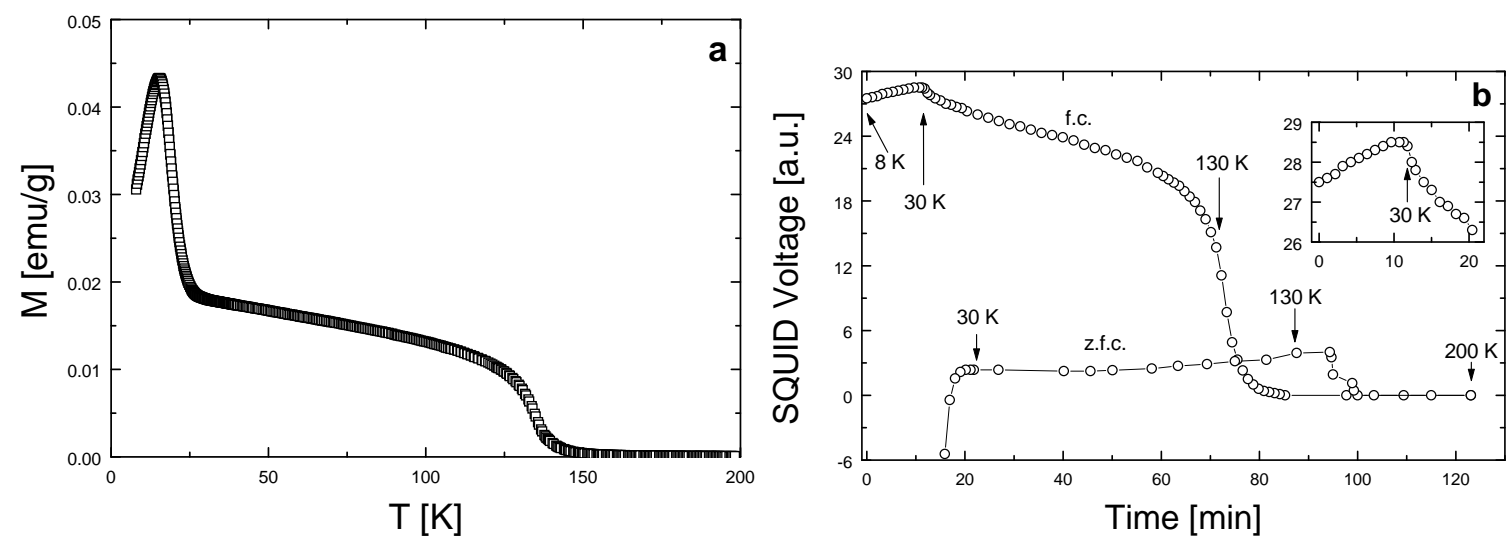

FIG. 8: (a) f.c. d.c. magnetization measurement of the moving Ru-1212 sample, where the peak like feature at low temperatures is obvious. The measurement was done in a nominal field of $0.25 \mathrm{mT}$. (b) The output voltage of the SQUID circuit with stationary sample in the same field. Characteristic temperatures given at certain time points are derived from a thermometer located in the flowing He gas a few centimeters away from the sample and should be considered as a guide to the eye. The data are corrected for the voltage vs. time drift of the SQUID setup. Inset: the low temperature part of the f.c. measurement.

during the measurement, when the magnetometer's field is not homogeneous. Libbrecht et al. 37 , using the Bean mode 38 , have shown that, depending on the sample properties and the level of field inhomogeneity, it is even possible, that the sample's magnetization will change sign and eventually reach its original value with reversed sign at the end of the scan in a non-homogeneous field. Such problems creating spurious signals in the d.c. magnetization of superconducting materials have been discussed in the past by several authors $31,36,37,39,40$.

The above analysis motivated us to investigate whether $\mathrm{Ru}-1212$, in its superconducting state, shows a similar sensitivity to field inhomogenieties, which could create artefacts in its measured magnetization. We worked in the following way: another cycle of the magnet from high magnetic fields to zero and finally to $0.25 \mathrm{mT}$ was done. After this cycle the peak in the f.c. d.c. magnetization data reappeared, as shown in figure 8a. After this measurement and without changing the field, we placed the sample in the center of the pickup coil system and recorded the output voltage of the SQUID circuit as the sample was cooled from $200 \mathrm{~K}$ without moving it. With this type of measurements absolute values of the magnetization can not be calculated, however, the recorded signal is proportional to 
the magnetization of the sample. The result is shown in figure $8 \mathrm{~b}$. No peak like feature is observed at low temperatures in this measurement. Only a decrease of the magnetization indicative of field expulsion as the sample is entering the superconducting state is observed. In the same figure we have also included the z.f.c. measurement for our sample. Again, no peak like feature of the magnetization at low temperatures is observed. The measurements presented in figure 8 clearly illustrate that the peak like features of the d.c. magnetization observed in the superconducting state of Ru-1212 are an experimental artefact arising from the movement of the sample in an inhomogeneous field during the measurement in the SQUID magnetometer.

In view of the observed sensitivity of the Ru-1212 measured magnetization to field inhomogenieties and since we have no evidence that a field reversal, similar to that for the measurements presented in figure 6, or cycling of the magnet, similar to that before the measurements presented in figure 7, affect the field profile of the superconducting magnet in a systematic way, we consider measurements similar to those in figure 8b as the most reliable test, that helps to decide, whether the observed features in a SQUID magnetization measurement of Ru-1212 (or any other superconductor) represent a true property of the compound or not.

\section{E. Some remarks for Sr-2116}

The field profile of the SQUID's superconducting magnet can affect the measured magnetization not only of superconducting samples (like Ru-1212) but also of any sample showing hysteresis in its magnetization. This sets the validity of the reported magnetization peak for Sr-2116 under question (see section [IIB and reference [28]). Thus, we tested Sr-2116 as introduced above. In figure 9 a we show a f.c. measurement of a Sr-2116 sample in a negative field of $-0.25 \mathrm{mT}$ and in figure $9 \mathrm{~b}$ a f.c. measurement of the SQUID setup's output voltage in a field of $0.25 \mathrm{mT}$. The peak, which has been attributed to a response of the paramagnetic Gd moments to the antiferromagnetic ordering of the $\mathrm{Ru}$ moments at about

$35 \mathrm{~K} 28$, is reversed in the negative field and is also obvious in in the measurement without moving the sample. These measurements show, that the reported 28 magnetization peak for Sr-2116 represents a true property of the material. 

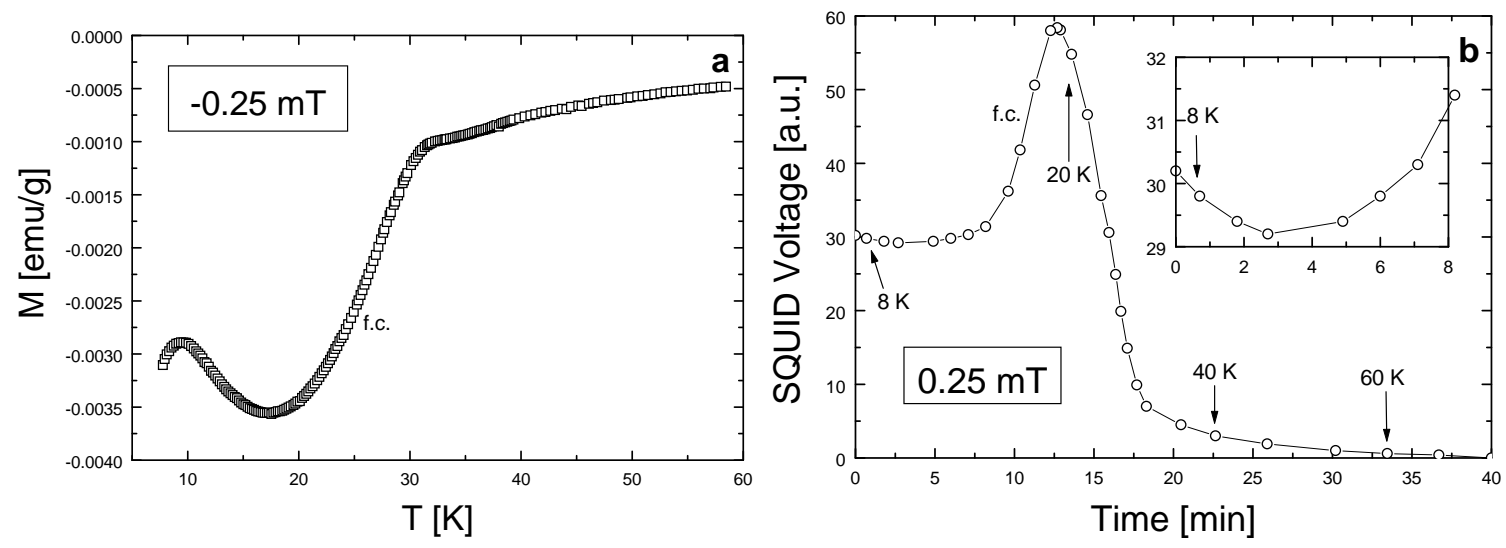

FIG. 9: (a) f.c. d.c. magnetization measurement of the moving Sr-2116 sample in a field of $0.25 \mathrm{mT}$. (b) The output voltage of the SQUID circuit with stationary sample in a field of $0.25 \mathrm{mT}$. Characteristic temperatures given at certain time points are derived from a thermometer located in the flowing He gas a few centimeters away from the sample and should be considered as a guide to the eye. The data are corrected for the voltage vs. time drift of the SQUID setup. Inset: the low temperature part of the f.c. measurement.

\section{CONCLUDING REMARKS}

In summary, we have investigated the origin of "paramagnetic"-like features (see figures 2 and 7) in the superconducting state of Ru-1212. We have shown, that these features, with high probability, are experimental artefacts arising from the movement of the sample in an inhomogeneous field during the measurement with the SQUID magnetometer. In view of the observed sensitivity of the Ru-1212 measured properties to the field profile of the superconducting magnet, we have proposed and evaluated several tests, which can help to decide whether the observed features in a SQUID magnetization measurement of $\mathrm{Ru}$ 1212 is an intrinsic property of the compound or not, with the most reliable one being the recording of the SQUID circuit's output voltage as the sample is cooled or warmed up in the magnetometer without being moved. We consider these tests as general tests, which can be used for the evaluation of the data on any superconducting sample.

Our work shows, that any "paramagnetic"-like features in the superconducting state of $\mathrm{Ru}-1212$, which could easily be related to some response of the Gd moments in this compound and considered as an indication for the lack of a Meissner state for Ru-1212, have 
to be evaluated carefully. On the other hand, features indicative of the existence of the Meissner state for this compound have also to be investigated carefully. Our work together with the measurements of McElfresh et al. 31 indicate, that any measured "paramagnetic"like features can easily be turned to "diamagnetic"-like features, which could be mistaken as an indication of the existence of a bulk Meissner state for Ru-1212, by a reversal of the field profile with respect to the set value of the magnetic field. The f.c. measurement on a stationary sample presented in figure $8 \mathrm{~b}$ indicates, that the Meissner state indeed exists for Ru-1212. Nevertheless, similar measurements, where the calculation of the absolute values of the magnetization will be possible for an estimation of the superconducting volume of the sample, are necessary, in order to clarify this issue.

Both here and in our previous work 28 we have shown, that Sr-2116 can also be a good candidate for the explanation of any "peculiar" features in the low temperature properties of Ru-1212 like d.c. magnetization and specific heat. Since new Ru-1212 compounds with other lanthanides in the place of Gd have recently been reported $17.18,19,41$, we note, that there is a whole series of Sr-2116 compounds also with other lanthanides in the place of Gd, which are magnetic at low temperatures, 43,44, 45, 46, 47. Some of these Sr-2116 compounds doped with $\mathrm{Cu}$, like $\mathrm{Sr}_{2} \mathrm{YRu}_{1-x} \mathrm{Cu}_{x} \mathrm{O}_{6}$ (but not $\mathrm{Sr}_{2} \mathrm{GdRu}_{1-x} \mathrm{Cu}_{x} \mathrm{O}_{6} 2$ ) are also reported superconducting

with a $T c$ similar to those of the Ru-1212 compounds 4 , 49, 50,51,52. Careful studies, similar to those in our previous work 28 and in section $1 \mathrm{IIB}$ are necessary to exclude the possibility, that trace impurities of Sr-2116 compounds are responsible for the properties attributed to the $\mathrm{Ru}-1212$ phase.

\section{Acknowledgments}

We would like to thank Dr. Alexander Schindler for useful discussions.

* Corresponding author: Thomas.Papageo@uni-bayreuth.de

1 J. G. Bednorz and K. A. Müller, Z. Phys. B 64, 189 (1986)

2 Y. Maeno, H. Hashimoto, K. Yoshida, S. Nishizaki, T. Fujita, J. G. Bednorz, and F. Lictenberg, Nature 372, 532 (1994)

3 J. Bardeen, L. N. Cooper, and J. R. Schrieffer, Phys. Rev. 108, 1175 (1957) 
4 For a review of the structural and superconducting properties of the HTSC compounds see, for example, M. Cyrot, and D. Pavuna, Introduction to superconductivity and high- $T_{c}$ materials, World Scientific (1992), or C. P. Poole, H. A. Farach, and R. J. Creswick, Superconductivity, Academic Press (1995) and references therein.

5 L. Bauernfeind, Supraleitung und Magnetismus in rutheniumhaltigen Kupraten, PhD thesis, Universität Bayreuth, (1998)

6 L. Bauernfeind, W. Widder, H. F. Braun, Physica C 254, 151 (1995)

7 L. Bauernfeind, W. Widder, and H. F. Braun, J. Low Temp. Phys. 105, Nos. 5/6, 1605 (1996)

8 L. Klein, J. S. Dodge, C. H. Ahn, J. W. Reiner, L. Mieville, T. H. Geballe, M. R. Beasly, and A. Kapitulnik, J. Phys.: Condens. Matter 8, 10111 (1996)

9 A. C. McLaughlin, W. Zhou, J.B. Attfield, A. N. Fitch, J. L. Tallon, Phys. Rev. B 60, 7512 (1999)

10 I. Felner, U. Asaf, S. Reich, Y. Tsabba, Physica C 311, 163 (1999)

11 C. Bernhard, J. L. Tallon, E. Brücher and R. K. Kremer, Phys. Rev. B 61, R14960 (2000)

12 P. W. Klamut, B. Dabrowski, S. Kolesnik, M. Maxwell, and J. Mais, Phys. Rev. B 63, 224512 $(2001)$

13 O. Chmaissem, J. D. Jorgensen, H. Shaked, P. Dollar, J. L. Tallon, Phys. Rev. B 61, 6401 (2000)

14 J. W. Lynn, B. Keimer, C. Ulrich, C. Bernhard, and J. L. Tallon, Phys. Rev. B 61, R14964 (2000)

15 J. D. Jorgensen, O. Chmaissem, H. Shaked, S. Short, P. W. Klamut, B. Dabrowski, and J. L. Talon, Phys. Rev. B 63, 054440 (2001)

16 C. Bernhard, J. L. Tallon, Ch. Niedermayer, Th. Blasius, A. Golnik, E. Brücher, R. K. Kremer, D. R. Noakes, C. E. Stronach and E. J. Ansaldo, Phys. Rev. B 59, 14099 (1999)

17 C. V. M. Williams and S. Krämer, Phys. Rev. B 62, 4132 (2000)

18 H. Takagiwa, J. Akimitsu, H. K. Furukawa and H. Yoshizawa, J. Phys. Soc. Jap. 70, 333 (2001)

19 D. P. Hai, S. Kamisawa, I. Kakeya, M. Furuyama, T. Mochiku, K. Kadowaki, Physica C 357360, 406 (2001)

20 L. Bauernfeind, W. Widder, H. F. Braun, FOURTH EURO CERAMICS, Vol. 6, 329 (1995)

21 I. Felner, U. Asaf, Y. Levi, and O. Milo, Phys. Rev. B 55, R3374 (1997)

22 S. Y. Chen, J. Shulman, Y. S. Wang, D. H. Cao, C. Wang, Q. Y. Chen, T. H. Johansen, W. K. 
Chu and C. W. Chu, cond-mat/0105510

23 Recently, coexistence of magnetism and superconductivity has also been reported for $\left(\mathrm{Fe}_{0.5} \mathrm{Cu}_{0.5}\right) \mathrm{SrBaYCu}_{2} \mathrm{O}_{7+\delta}$ by: Z. A. Ren, G. C. Che, H. Xiong, K. Q. Li, Y. S. Yao, D. N. Zheng, Y. M. Ni, S. L. Jia, C. Dong, H. Chen, J. L. Shen, Z. X. Zhao, Sol. St. Com. 119, $579(2001)$

24 Y. Y. Xue, R. L. Meng, J. Cmaidalka, B. Lorenz, L. M. Desaneti, A. K. Heilman and C. W. Chu, Physica C 341-348, 459 (2000)

25 C.W. Chu, Y. Y. Xue, S. Tsui, J. Cmaidalka, A. K. Heilman, B. Lorenz, R. L. Meng, Physica C 335, 231 (2000)

26 J. L. Tallon, J. W. Loram, G. V. M. Williams, C. Bernhard, Phys. Rev. B 61, R6471 (2000)

27 A. Fainstein, E. Winkler, A. Butera, J. Tallon, Phys. Rev. B 60, R12597 (1999)

28 T. P. Papageorgiou, T. Herrmannsdörfer, R. Dinnebier, T. Mai, T. Ernst, M. Wunschel and H. F. Braun, cond-mat/0205462 and Physica C, in press

29 P. W. Klamut, B. Dabrowski, M. Maxwell, J. Mais, O.Chmaissem, R. Kruk, R. Kmiec, and C. W. Kimball, Physica C 341-348, 455 (2000)

30 C. Artini, M. M. Carnasciali, G. A. Costa, M. Ferretti, M. R. Cimberle, M. Putti and R. Masini, Physica $\mathrm{C}$, in press

31 M. McElfresh, S. Li and R. Sager, Effects of Magnetic Field Uniformity on the Measurement of Superconducting Samples, Quantum Design (San Diego), technical report (1996)

32 W. Widder, L. Bauernfeind, H. F. Braun, H. Burkhardt, D. Rainer, M. Bauer, H. Kinder, Phys. Rev. B 55, 1254 (1997)

33 G. F. Zharkov, Phys. Rev. B 63, 214502 (2001)

34 J. Horvat, X. L. Wang, S. Soltanian and S. X. Dou, cond-mat/0201006

35 E. B. Sonin, I. Felner, Phys. Rev. B 57, R14000 (1998)

36 F. J. Blunt, A. R. Perry, A.M. Campbell and R. S. Liu, Physica C 175, 539 (1991)

37 S. Libbrecht, E. Osquiguil, Y. Bruynseraede, Physica C 225, 337 (1994)

38 C. P. Bean, Rev. Mod. Physics, 31 (1964)

39 A. Schilling, H. R. Ott, Th. Wolf, Phys. Rev. B 46, 14253 (1992)

40 W. Braunisch, N. Knauf, V. Kataev, S. Neuhausen, A. Grütz, A. Kock, B. Roden, D. Khomskii and D. Wohlleben, Phys. Rev. Lett. 68, 1908 (1992)

41 R. Ruiz-Bustos, J. M. Gallardo-Amores, R. Sáez-Puche, E. Morán and M. Á. Alario-Franco, 
Physica C, in press

42 P. D. Battle, J. B. Goodenough, and R. Price, J. Solid State Chem. 46, 234 (1983)

43 P. D. Battle and W. J. Macklin, J. Solid State Chem. 52, 138 (1984)

44 P. D. Battle and W. J. Macklin, J. Solid State Chem. 54, 245 (1984)

45 P. D. Battle and C. W. Jones, J. Solid State Chem. 78, 108 (1989)

46 P. D. Battle, C. W. Jones, and F. Studer, J. Solid State Chem. 90, 302 (1991)

47 Y. Doi and Y. Hinatsu, J. Phys.: Condens. Matter 114813 (1999)

48 M. K. Wu, S. R. Sheen, D. C. Ling, C. Y. Tai, G. Y. Tseng, D. H. Chen, D. Y. Chen, F. Z. Chien, and F. C. Zhang, Chech. J. Phys. 46 (Suppl. S6), 3381 (1996)

49 D. Y. Chen, F. Z. Chien, D. C. Ling, J. L. Tseng, S. R. Sheen, M. J. Wang, and M. K. Wu, Physica C 282-287, 73 (1997)

50 M. K. Wu, D. Y. Chen, F. Z. Chien, S. R. Sheen, D. C. Ling, C. Y. Tai, G. Y. Tseng, D. H. Chen, and F. C. Zhang, Z. Phys. B 102, 37 (1997)

51 H. A. Blackstead, John D. Dow, D. R. Harshman, M. J. DeMarco, M. K. Wu, D. Y. Chen, F. Z. Chien, D. B. Pulling, W. J. Kossler, A. J. Greer, C. E. Stonach, E. Koster, B. Hitti, M. Haka, and S. Toorongian, Eur. Phys. J. B 15, 649 (2000)

52 M. K. Wu, D. Y. Chen, D. C. Ling, and F. Z. Chien, Physica B 284-288, 477 (2000) 